\title{
Condicionantes e características da mortalidade materna no Brasil
}

\author{
Conditions and characteristics of maternal mortality in Brazil
}

Condiciones y características de la mortalidad materna en Brasil

\begin{abstract}
Matheus Serapião Teodoro ${ }^{*}$, Pedro Henrique Elias dos Santos ${ }^{1}$, Mirelle Caroline de Souza ${ }^{1}$, Diogo Bohon Riskalla1, João Vitor Borges Barbosa1, Bárbara Delucca Gatti Guimarães", Michael Zarnowski Passos¹.
\end{abstract}

\section{RESUMO}

Objetivo: Abordar os aspectos relacionados à mortalidade materna (MM) no Brasil e discutir a implementação de medidas úteis para sua redução. Métodos: Trata-se de um artigo original, baseado em estudos em inglês e português, sobre a MM no Brasil e em outros países, publicados entre 2008 e 2021. Os estudos foram selecionados nas plataformas PubMed, SciELO, Acervo+ e Medline, aplicando os descritores: Mortalidade Materna, Gravidez de Alto Risco, Serviço de Saúde da Mulher. Ademais, foram utilizados boletins epidemiológicos e sites do Ministério da Saúde (MS) e da Organização Mundial da Saúde (OMS). Posto que as informações são de domínio e acesso público, a aprovação do comitê de ética e pesquisa é dispensável. Resultados: A MM possui causas diretas e indiretas e está intimamente relacionada aos condicionantes sociais, explicitando agravos no acesso à saúde e à educação e a acentuada presença de uma baixa condição socioeconômica no Brasil. A persistência da subnotificação no país e fatores como raça, condição civil, tipo de parto, idade e escolaridade influenciam a MM. Conclusão: Embora a MM tenha apresentado uma atenuação nos últimos anos, ainda apresenta índice elevado no Brasil, evidenciando a importância da adoção de medidas que visem a sua redução.

Palavras-chave: Mortalidade materna, Gravidez de alto risco, Serviço de saúde da mulher.

\begin{abstract}
Objective: To address aspects related to maternal mortality (MM) in Brazil and discuss the implementation of useful measures for its reduction. Methods: This is an original article based on studies in English and Portuguese, about MM in Brazil and other countries, published between 2008 and 2021. The studies were selected on the PubMed, SciELO platforms, Acervo + and Medline, applying the descriptors: Maternal mortality, High-Risk Pregnancy, Women's Health Services. In addition, epidemiological bulletins and the Ministry of Health (MS) and the World Health Organization (WHO) websites were used. As they are public domain and accessible information, the approval of the Research Ethics Committee is expendable. Results: The MM can have direct or indirect causes and is closely related to social conditions, demonstrating worsening access to health and education and the marked presence of a low socioeconomic condition in Brazil. The persistence of underreporting in the country, in addition to race, marital status, mode of delivery, age and school education influence the MM. Conclusion: Although the maternal mortality rate has presented an attenuation in the past few years, it is still high in Brazil, showing the importance of adopting a series of actions aimed at mitigating it.
\end{abstract}

Keywords: Maternal mortality, High-risk pregnancy, Women's health services.

\section{RESUMEN}

Objetivo: Abordar aspectos relacionados con la mortalidad materna (MM) en Brasil y discutir la implementación de medidas útiles para su reducción. Métodos: Se trata de un artículo original basado en estudios en inglés y portugués, sobre MM en Brasil y otros países, publicados entre 2008 y 2021. Los estudios fueron seleccionados en las plataformas PubMed, SciELO, Acervo + y Medline, aplicando los descriptores: Mortalidad materna, Embarazo de Alto Riesgo, Servicios de Salud para Mujeres. Además, se utilizaron boletines epidemiológicos y sitios web del Ministerio de Salud (MS) y la Organización Mundial de la Salud (OMS). Como son información de dominio y de acceso público, es prescindible la aprobación del comité de ética de la investigación. Resultados: La MM puede tener causas directas o indirectas y está estrechamente relacionado con las condiciones sociales, lo que demuestra un empeoramiento del acceso a la salud y la educación y la marcada presencia de una condición socioeconómica baja. La persistencia de subregistro en el país, además de características como raza, estado civil, modo de parto, edad y enseñanza influyen en la MM. Conclusión: Aunque la razón de la mortalidad materna ha mostrado una atenuación en los últimos años, sigue siendo alta en Brasil, lo que destaca la importancia de adoptar medidas encaminadas a mitigarla.

Palabras clave: Mortalidad materna, Embarazo de alto riesgo, Servicios de salud para mujeres.

${ }^{1}$ Faculdade de Minas (FAMINAS-BH), Belo Horizonte - MG. *E-mail: matheussteodorolp@gmail.com 


\section{INTRODUÇÃO}

A Mortalidade Materna (MM) é definida pela Organização Mundial da Saúde (OMS) como a morte de uma mulher durante a gestação, ou dentro de um período de até 42 dias após o término desta, devido a qualquer causa relacionada ou agravada pela gravidez ou por medidas relacionadas a ela, todavia, não por causas acidentais ou incidentais (BRASIL, 2020).

A MM é dividida em causas diretas e indiretas, sendo que, a primeira engloba complicações obstétricas, durante a gravidez, parto ou puerpério, alusivas às intervenções, omissões ou tratamento incorreto (Hipertensão, embolia, hemorragia, infecção puerperal, Infecções do trato urinário (ITU) na gestação, aborto, complicações relacionadas ao trabalho de parto, entre outros). Em contrapartida, as causas indiretas são aquelas que resultam no agravamento de doenças de base ou que se desenvolveram durante o período da gravidez, não provocadas por causas obstétricas (doenças do aparelho circulatório e respiratório, Vírus da imunodeficiência humana/Síndrome da imunodeficiência adquirida (HIV/AIDS), Hipertensão pré-existente, transtornos mentais, etc) (CARVALHO PI, et al., 2020).

Além de se destacar como um importante tópico da saúde pública, a MM é um bom marcador do desenvolvimento de um país, capaz de denunciar desigualdades sociais, problemas socioeconômicos e falta de assistência em saúde (SCARTON J, et al., 2019).

É estimada, globalmente, uma razão de mortalidade materna (RMM) de 216 para cada 100 mil nascidos vivos, porém, ao se observar os dados de países em diferentes estratos socioeconômicos, torna-se notável a grande disparidade. Nos países desenvolvidos, é estimada uma RMM de cerca de 12 para cada 100 mil nascidos vivos (NV), enquanto que nos países em desenvolvimento essa taxa é estimada em 239 para cada 100 mil NV (WHO, 2015). A África Subsaariana, por exemplo, sozinha, contempla cerca de $66 \%$ do total global de mortes maternas, com uma RMM que alcança 546 para cada 100 mil nascidos vivos nessa região, em contrapartida, no Brasil, essa taxa é de cerca de 60 para cada 100 mil NV (WHO, 2015 e BRASIL, 2020).

Apesar dos esforços, o Brasil tem falhado no que tange ao combate da MM, pois mantém parâmetros superiores aos propostos pela ONU e pelos acordos entre países (20 e 30-35 mortes por 100 mil NV, respectivamente). Grande parte da dificuldade em alcançar esse objetivo se dá pela significativa importância dos fatores sociais (raça, escolaridade, acesso à saúde, estado civil, etc) vinculados a esse tema. Ante o exposto, faz-se visível a necessidade de políticas públicas de cunho social, que ampliem o acesso à saúde e promovam melhorias na qualidade dos serviços ofertados, atenuando o abismo socioeconômico, a fim de que os índices de MM sejam reduzidos (MARTINS ACS e SILVA LS, 2018).

O objetivo desse estudo foi mostrar a situação do Brasil, no que tange à MM, frente ao cenário mundial, e compreender o motivo da sua posição, abordando os fatores influenciadores e sua evolução ao longo dos últimos anos, além de traçar possíveis medidas de combate ao crescimento desse índice.

\section{MÉTODOS}

Para a produção do presente trabalho foram utilizados artigos em inglês e português, sobre MM no Brasil e em outros países. Os artigos utilizados como base foram preferencialmente selecionados nas plataformas de pesquisa em saúde; PubMed da National Library of Medicine (NLM), Scientific Eletronic Library Online (SciELO), Acervo+ e Medical Literature Analysis and Retrieval System Online (MEDLINE). Ademais, foram utilizados guias e boletins de vigilância epidemiológicos e os sites do MS e da OMS. As palavras-chave selecionadas como guias para a coleta de dados foram: Mortalidade Materna; Gravidez de Alto Risco; Serviço de Saúde da Mulher.

O período abordado pela pesquisa de artigos, especificamente, consistiu no intervalo entre os anos de 2008 e 2020, permitindo uma abordagem aprofundada a respeito do tema, por intermédio de diferentes percepções e mudanças do perfil epidemiológico ao longo desse período.

Esse trabalho se trata de uma produção original que teve como base informações de domínio público, passíveis de acesso em sites do MS. Assim, pelo fato das informações aqui contidas serem de origem pública, é dispensada a aprovação do Comitê de Ética em Pesquisa (CEP). 


\section{RESULTADOS e DISCUSSÃO}

A MM é considerada uma violação grave dos direitos humanos das mulheres, graças ao fato da mesma ser um evento de fato evitável na imensa maioria das vezes (85-95\%), visto que as causas diretas são consideradas, em sua quase totalidade, um problema de passível prevenção (VIANA RC, et al., 2011; BRASIL, 2009). Contudo, mesmo diante do exposto a pouco, a MM ocorre com peculiaridades desiguais, ao se comparar países desenvolvidos, subdesenvolvidos e em desenvolvimento, especialmente nos 2 últimos citados, em que atinge níveis alarmantes e inaceitáveis. Tal fato manifesta claramente que existe uma íntima relação da MM com a situação socioeconômica dos países estudados (FALCÃO NHF, et al., 2011). A RMM é o indicador utilizado para mensurar a MM através da fórmula (Quadro 1).

Quadro 1 - Fórmula utilizada para o Cálculo da RMM.

$\mathrm{MM}=$ (№ de óbitos de mulheres por causas ligadas à gravidez,parto e puerpério $\div$ №
de nascidos vivos $) \times 100.000 "$

Fonte: Teodoro MS, et al., 2021; dados extraídos do Boletim epidemiológico nํ20 do Ministério da Saúde (MS), 2020.

Há tempos, essa questão é colocada como pauta de discussão em reuniões mundiais sobre saúde, por configurar uma importante questão de saúde pública, além de se mostrar um indicador confiável do desenvolvimento socioeconômico de um país, de tal forma que diversos programas e acordos foram firmados entre países, como o "Objetivos de Desenvolvimento do Milênio" (ODM), estipulado pela organização das nações unidas e pactuado pelo Brasil nos anos 2000. Esse programa teve como meta reduzir a RMM nacional para pelo menos 35 óbitos por 100 mil NV até 2015. Hodiernamente, verifica-se a presença do Brasil no programa "Objetivos de Desenvolvimento Sustentável" (ODS), cujo desejo é reduzir, até 2030, a RMM para 30 óbitos para cada 100 mil NV (BRASIL, 2020; NOUR NM, 2008; FALCÃO NHF, et al., 2001).

Para definir um óbito como pertencente ao grupo da MM é necessária uma investigação minuciosa, que tem início na identificação correta do óbito, seguido da coleta de dados por meio de entrevistas no domicílio, entrevistas com os profissionais de saúde envolvidos no caso e, por último, uma pesquisa no registro de serviço de saúde (BRASIL, 2020).

Conforme já mencionado, a MM sofre uma forte influência de condicionantes sociais e, portanto, os níveis da MM são exponencialmente mais altos nos países subdesenvolvidos e em desenvolvimento, em razão da desigualdade social instalada nesses países, além de um alto número de mulheres com fatores que contribuem para uma acentuada evolução de tal desfecho em determinadas classes (SCARTON J, et al., 2019). O Brasil, em 2018, apresentou uma RMM de 59,1 óbitos por 100 mil NV (BRASIL, 2020), nível muito superior à 20 óbitos, que é o número considerado razoável pela Organização das Nações Unidas, ou mesmo os 30 óbitos estipulados na ODS. Para fins comparativos, países desenvolvidos apresentam uma média próxima a 10 óbitos por 100 mil NV, sendo ela quase um sexto dos números presentes na realidade brasileira (Figura 1) (MARTINS ACS e SILVA LS, 2018).

Figura 1 - RMM brasileira em relação às metas da ODM e ODS.

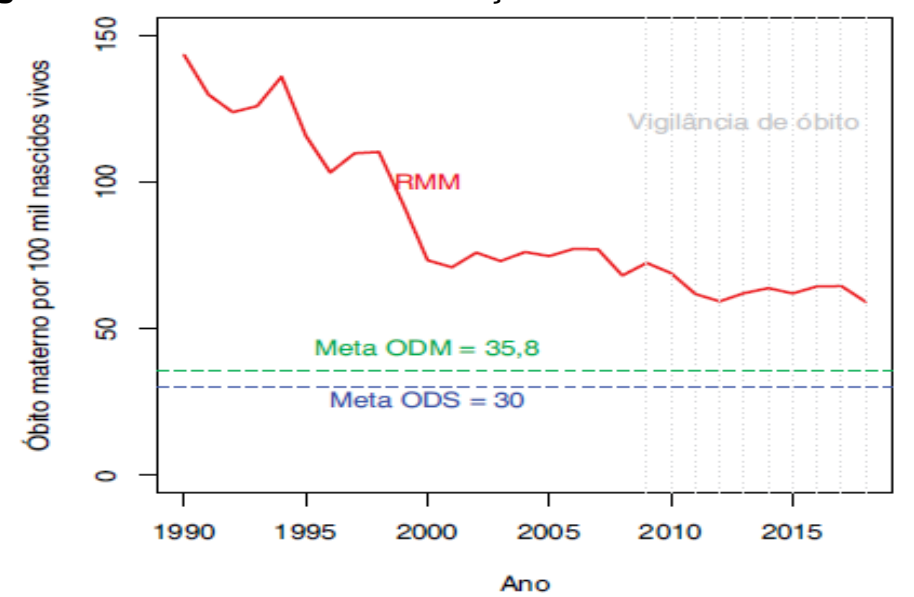

Fonte: Teodoro MS, et al., 2021; dados extraídos do Boletim epidemiológico №20 do Ministério da Saúde (MS), 2020. 
O Brasil apresentou uma queda de quase 10\% da RMM entre 2017 e 2018, passando de quase 65 óbitos para 59 óbitos por 100 mil nascidos vivos. Comparando o desenvolvimento dos estados em torna dessa mesma temática e no período acima referido, as regiões Norte, Nordeste e Sudeste lideraram com uma redução expressiva da RMM, mas ainda se mantendo na faixa de mais de 50 óbitos por 100 mil NV (Figura 2).

Figura 2 - Evolução da RMM nos estados brasileiros ao longo dos anos.
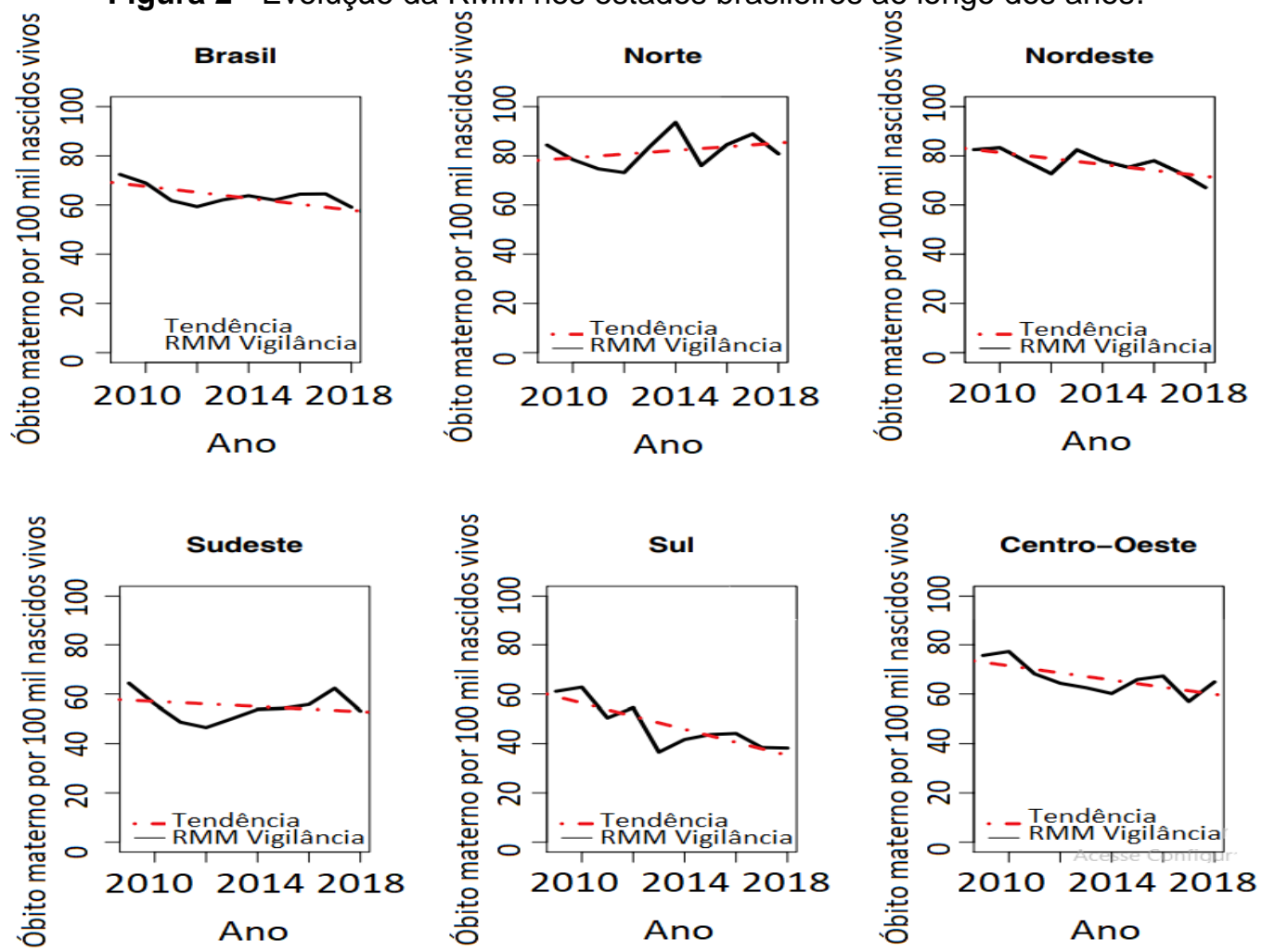

Fonte: Teodoro MS, et al., 2021; dados extraídos do Boletim epidemiológico ㄲo20 do Ministério da Saúde (MS), 2020.

Averiguando os níveis da RMM, com o passar dos anos, especialmente no intervalo entre 2009-2018, notase um avanço evidente, o qual provavelmente pode ser atribuído a uma melhoria nas investigações, a uma ampliação do acesso à saúde, às medidas e programas sociais que beneficiaram os grupos mais afetados, a melhoria da qualidade da saúde ofertada, etc. Além disso, é importante mencionar que de 1990 a 2008, o Brasil apresentou seu maior sucesso na redução da MM, tendo alcançado uma queda de pouco mais de $50 \%$ na RMM (WHO, 2010).

Outrossim, é importante mencionar que as questões socioeconômicas se mantêm como um fator determinante, ao se comparar a RMM entre os estados brasileiros, visto que as regiões Norte e Nordeste apresentam-se com os maiores valores de MM, o que demonstra, mais uma vez, a discrepância existente, no que tange ao acesso à saúde e à educação, e presença forte de uma baixa condição socioeconômica dentre outros fatores importantes (MORSE ML, et al., 2011; CARVALHO PI et al., 2020). Entretanto, alguns artigos demonstraram ainda que existem vários países com uma situação socioeconômica aquém à brasileira, como Chile, Uruguai, Cuba, que detém uma RMM semelhante às taxas de países desenvolvidos, tal fato, explana que a qualidade do atendimento à mulher é um fator corretivo essencial desse desfecho, mesmo quando a questão socioeconômica é desfavorável (VIANA RC, et al., 2011).

Em relação às causas da morte materna, como mencionado, a imensa maioria, representando cerca de $90 \%$, são evitáveis. Sendo que as causas diretas respondem por $2 / 3$ do total de mortes em países subdesenvolvidos e em desenvolvimento, enquanto as causas indiretas são a maioria nos países desenvolvidos (SANTOS DR, et al., 2017) (Figura 3). Há uma certa divergência na literatura, sobre qual a principal causa de morte materna, no entanto, existe uma predominância de dados que apontam os distúrbios 
hipertensivos como a principal causa de morte materna, inclusive no Brasil, seguido então por distúrbios hemorrágicos, infecções e aborto (Figura 4) que, segundo dados da International Federation of Gynecology and Obstetrics (FIGO) representam cerca de 10\% das mortes maternas (VIANA RC, et al., 2011; MORSE ML, et al., 2011; BRASIL, 2020; RUAS CAM, et al., 2020; CARVALHO PI, et al., 2020).

Existem ainda dados apontando o fato de que a maioria das mortes acontecem no período puerperal, enquanto outros, em contrapartida, afirmam que a maioria das mortes ocorrem no período gestacional (CARVALHO PI, et al., 2020; CORTINHAS ABB, et al., 2019; SCARTON J, et al., 2019). Tais registros mostram, portanto, a importância em melhorar e, especialmente, prolongar o período de acesso da atenção e suporte durante o período gravídico-puerperal, frente a constatação de que a maioria das mulheres tem um pré-natal deficitário, em algum momento, com acompanhamento insatisfatório e com frequentes falhas assistenciais (VIANA RC, et al., 2011; SANTOS DR, et al., 2017; SCARTON J, et al., 2019).

Figura 3 - O comparativo entre causas diretas e indiretas de mortalidade materna no Brasil.

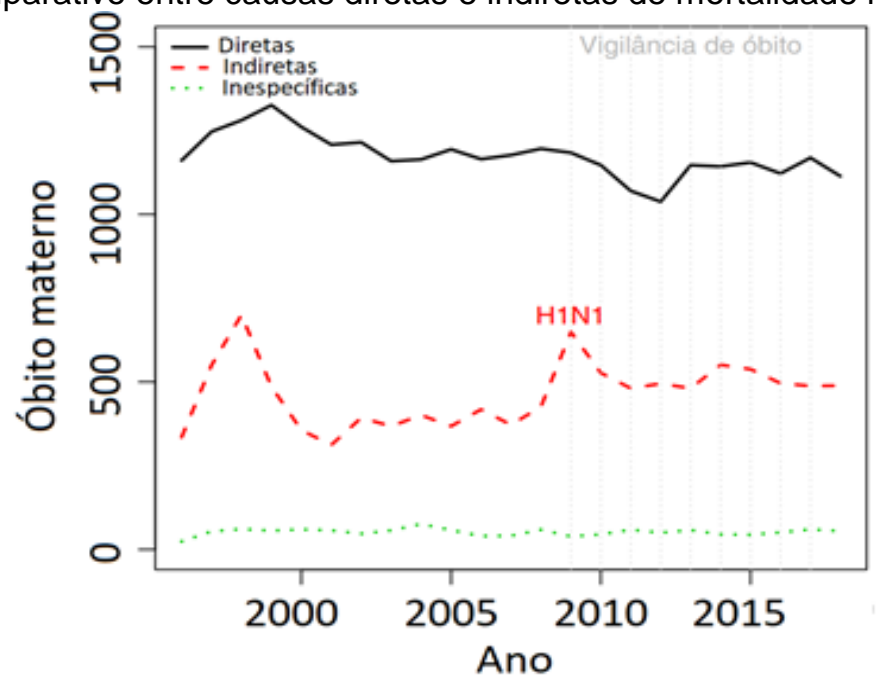

Fonte: Teodoro MS, et al., 2021; dados extraídos do Boletim epidemiológico ㄲo20 do Ministério da Saúde (MS), 2020.

Figura 4 - Causas de morte materna e sua prevalência no total de casos.
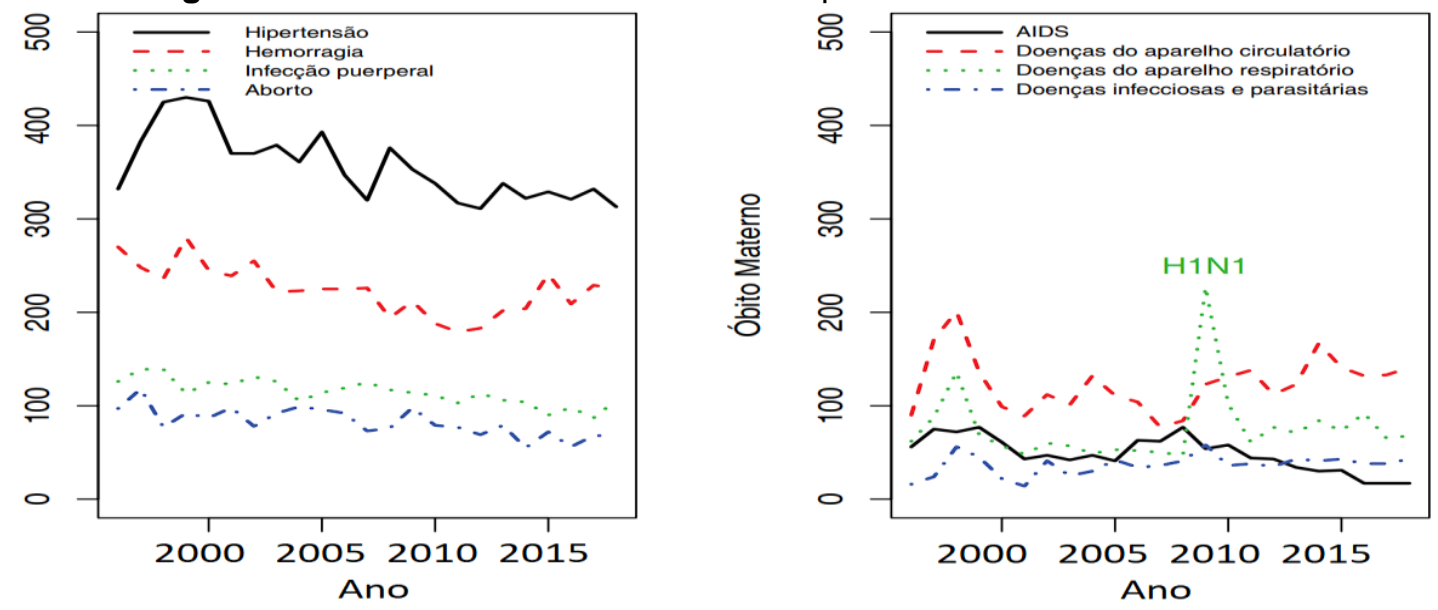

Fonte: Teodoro MS, et al., 2021; dados extraídos do Boletim epidemiológico no20 do Ministério da Saúde (MS), 2020.

Desse modo, faz-se necessária uma avaliação detalhada dos vários fatores que denotam maior ligação com a $\mathrm{MM}$ e os seus porquês, já que a RMM no Brasil ainda não alcançou os parâmetros desejados pela OMS, evidenciando-se, portanto, o cenário brasileiro pertinente a esta temática e as potenciais áreas em que há condições deficitárias passíveis de intervenções resolutivas. Dentre eles, destacam-se alguns, os quais serão elucidados neste trabalho. 
Por mais que os valores da RMM brasileira divulgados já sejam alarmantes, a situação é, provavelmente, ainda pior, pois dados sugerem a existência de uma forte subnotificação da MM no país (FALCÃO NHF, et al., 2011; VICTORA GC, et al., 2011; VIANA RC, et al., 2011). Isso se deve, principalmente, à deficiência de investigação e à falta de um registro confiável dos óbitos maternos, gerando um quadro de penumbra de informação à tentativa de definição numérica e qualitativa do tema.

O problema da subnotificação decorre especialmente do preenchimento incorreto da declaração de óbito, ou mesmo da ausência de algumas informações no documento, sendo extremamente observados; omissão da relação entre a causa da morte e a gestação, parto ou puerpério, e falta de registro do óbito nos cartórios municipais. Essa conjuntura é frequentemente mais observada em estados com condições socioeconômicas de notória precariedade como Norte, Centro-Oeste e Nordeste, uma vez que em tais locais, faz-se mais comum a existência de cemitérios irregulares e informação popular deficitária sobre a importância da declaração de óbito para a epidemiologia do país (SCARTON J, et al., 2019; MARTINS ACS, et al., 2018; CARVALHO PI, et al., 2020; BRASIL, 2009).

Sob outra perspectiva, nos últimos anos, esse problema vem sendo combatido com grande eficácia, segundo dados do boletim epidemiológico de 2020, por exemplo, que mostrou que $91 \%$ dos óbitos de mulheres na idade fértil foram investigados em 2018, uma evolução comparada aos apenas $55 \%$ que foram observados em 2009 (Figura 5) (BRASIL, 2020).

Figura 5 - Relação dos estados no combate à subnotificação.

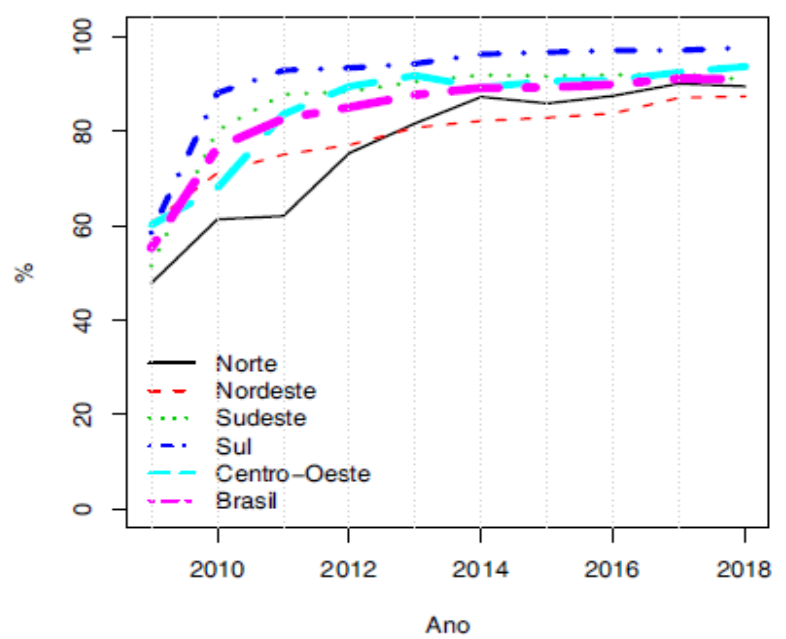

Fonte: Teodoro MS, et al., 2021; dados extraídos do Boletim epidemiológico nํ20 do Ministério da Saúde (MS), 2020.

A MM, de forma unânime, é maior em mulheres não brancas. Estudos nos Estados Unidos mostraram números que eram cerca de 3 a 4 vezes maiores, a respeito da mortalidade materna, ao se comparar negras com brancas. No Brasil, ainda que exista uma maior dificuldade em determinar o peso da raça, devido à grande miscigenação do país, o resultado não é diferente, mulheres pardas e negras configuram a maioria no número total de MM. Ademais, há estudos brasileiros que demonstraram uma diferença significativa na $\mathrm{MM}$ entre mulheres indígenas e não indígenas, de forma que as primeiras apresentaram uma RMM equivalente ao dobro das não indígenas (BRASIL, 2020; FALCÃO NHF, et al., 2011; SANTOS DR, et al., 2017).

O fato de a raça negra e parda estarem ligadas à maioria dos casos se deve a diversos aspectos, sendo os principais a maior prevalência de patologias hipertensivas, uma grande dificuldade de acesso à saúde, uma menor qualidade das assistências prestadas, uma situação econômica desfavorável, a maior prevalência do tabagismo e a alta prevalência de violência doméstica (CARVALHO PI, et al., 2020).

Infere-se, portanto, que o racismo estrutural é uma notável causa, que agrava a disparidade de mortes ao se comparar mulheres de diferentes cores, visto que as mulheres brancas morrem menos que as negras e pardas. Outra característica intrigante que foi observada é a condição conjugal, em que mulheres solteiras 
correspondem a maioria no total da MM, sendo esse dado passível de ser atribuído à falta de apoio emocional, afetivo, social, financeiro e estímulo ao autocuidado das mulheres, que se tornam mais vulneráveis a tais índices (SCARTON J, et al., 2019).

Por esse enfoque, faixas etárias extremas, sendo elas demasiadamente novas ou em idade avançada, são ligadas firmemente à maior chance de evolução para morte materna (VIANA RC, et al., 2011). Contudo, a maioria absoluta dos casos se fazem presentes na faixa etária de 20 a 35 anos, meramente graças a uma maior incidência da gravidez nessa faixa. (CORTINHAS ABB, et al., 2019; CARVALHO PI, et al., 2020; RUAS CAM, et al., 2020).

O nível de escolaridade também foi um fator avaliado na prevalência da $\mathrm{MM}$, constatando-se que mulheres com baixa escolaridade são ligadas ao aumento das chances de morte materna, possivelmente devido a influência do pouco entendimento sobre sinais e comportamentos de risco, do comportamento de não seguir instruções médicas, e pelo desconhecimento na identificação de um problema, quando presente, em tempo hábil (Figura 6) (VIANA RC, et al., 2011; SCARTON J, et al., 2019).

Ademais, outros fatores que foram identificados na maioria dos casos de morte materna foram a multiparidade e a cirurgia cesárea como forma de parto. Atribui-se a essa cirurgia o risco aumentado de infecção, tromboembolismo puerperal, complicações anestésicas e hemorrágicas (SCARTON J, et al., 2019; VIANA RC, et al., 2011; FEITOSA-ASSIS Al, et al., 2020).

Figura 6 - Condicionantes sociais e sua relação com a Mortalidade Materna.

Idade

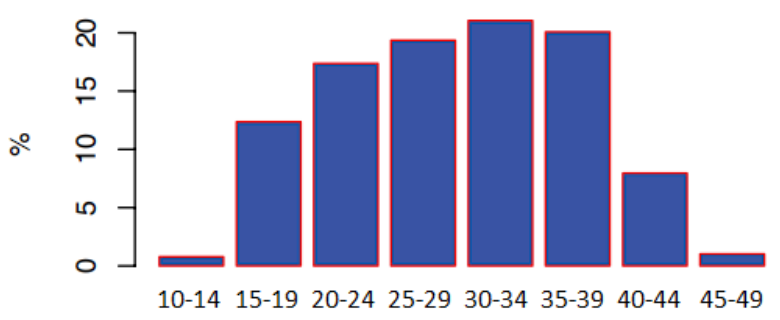

Estado Civil

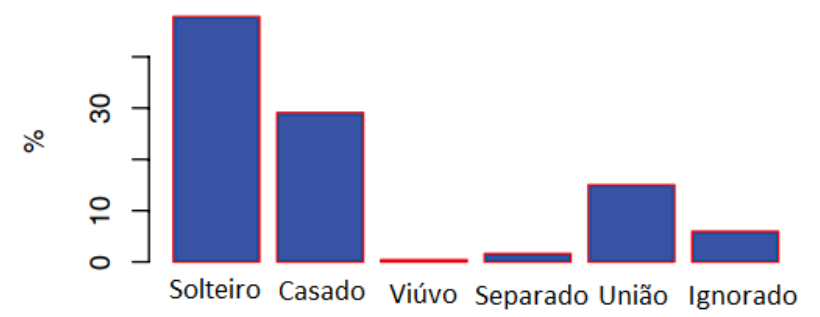

Raça/Cor

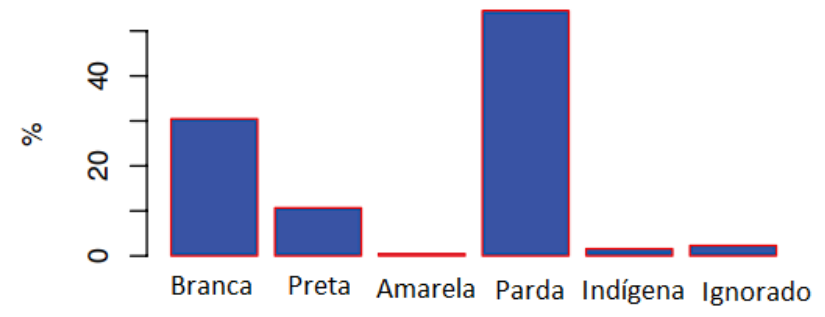

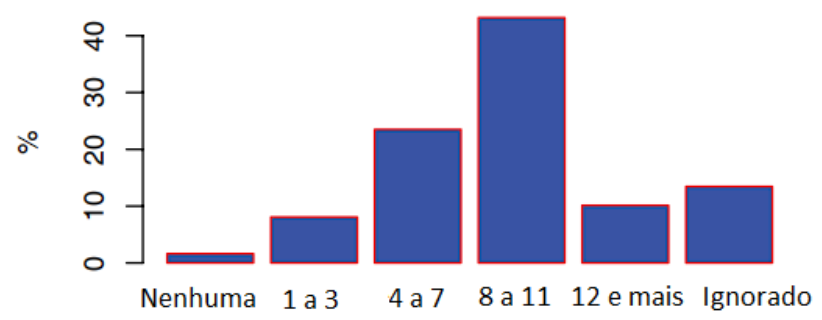

Fonte: Teodoro MS, et al., 2021; dados extraídos do Boletim epidemiológico nำ20 do Ministério da Saúde (MS), 2020.

Dessarte, é visível a necessidade de medidas que visem a redução da MM no país, podendo pontuar medidas de prevenção, diagnóstico precoce, tratamento adequado, aprimoramento e extensão do pré-natal, de cuidados no parto e puerpério, a fim de ampliar seu alcance, de forma a melhorar o planejamento familiar e 0 atendimento individualizado, humanizado e multidisciplinar da mulher. Adotar também medidas que diminuam a discrepância no acesso à saúde e educação, que visem investimentos de cunho social para diminuir a desigualdade socioeconômica que existe no país, que busquem combater o racismo estrutural e provocar melhoria na estrutura assistencial como um todo.

De forma suplementar, a respeito das condições sociais discutidas, torna-se evidente a complexidade da temática e a possibilidade de se evitarem desfechos indesejados na gestação, no que tange a $\mathrm{MM}$, ao se atentar e promover medidas públicas e socioeducativas, considerando o cenário desigual da sociedade 
brasileira. A atenção primária a saúde, nesse sentido, é realçada, mais uma vez, como um importante pivô, ao propiciar um ambiente de acompanhamento da gestação, assim como para medidas preventivas e educação em geral quanto à saúde das mulheres, abordando assuntos como o planejamento familiar e a educação em saúde sexual e reprodutiva.

É importante que a subnotificação seja abordada, melhorando o sistema de informação, tal como a fiscalização de documentos preenchidos de forma incorreta, deve-se ainda ampliar a investigação das causas de óbitos de mulheres em idade fértil, preencher de forma completa e correta a Declaração de Óbito, identificando a causa da morte em campos específicos.

É possível, também, haver algum benefício ao se intensificar a discussão sobre uma possível descriminalização do aborto, uma vez que o mesmo é um evento de grande frequência e, assim como outras causas de mortalidade materna, apresenta um certo grau de subnotificação, correspondendo a uma parcela maior da morbimortalidade materna em relação ao que se encontra em dados oficiais. Alguns estudos mostraram que um valor de aproximadamente 10\% das mortes maternas em 2002 resultou de práticas relacionadas ao aborto e que, no mesmo ano, 22\% das mulheres entre 35-39 anos reportaram que já haviam induzido um aborto (NOUR NM, 2008; RUAS CAM, et al., 2020; VICTORA GC, et al., 2011).

Em suma, é necessário que se invista em uma equipe multidisciplinar, desenvolvendo uma melhoria na orientação e capacitação profissional a respeito de medidas assistenciais, a fim de evitar um tratamento incorreto, ou mesmo a ausência do mesmo. Ainda, deve se fornecer, de forma apropriada, materiais necessários para possíveis intervenções, ampliar a disponibilidade de equipamentos, de bancos de sangue e de uma infraestrutura laboratorial adequada ao diagnóstico e tratamento dos casos das gestações de alto risco. O intuito deve se basear em desenvolver profissionais capacitados e equipados de forma satisfatória, que por fim evitarão complicações, que quando presentes serão tratadas em tempo hábil.

\section{CONCLUSÃO}

Observa-se que fatores como condição socioeconômica baixa, populações negra, indígena e parda, baixa escolaridade e mulheres solteiras, constituem grupos de risco associados a uma maior RMM, bem como mulheres submetidas a partos cesáreos. O Brasil, como um país em desenvolvimento, no qual uma parcela expressiva da população se encontra em situações de vulnerabilidade, associado às deficiências no acesso à saúde e às falhas nas investigações e notificações dos casos de MM, apresenta índices precários e preocupantes desse indicador. É imprescindível, então, a adoção de estratégias de diagnóstico precoce e planejamento familiar mais efetivas, melhoria da qualidade e do alcance da assistência pré-natal e ao parto e puerpério, e combate a subnotificação, de modo a alcançar melhores índices da RMM.

\section{REFERÊNCIAS}

1. BRASIL. MINISTÉRIO DA SAÚDE. Secretaria de Vigilância em Saúde. Departamento de Análise de Situação em Saúde. Guia de vigilância epidemiológica do óbito materno / Ministério da Saúde, Secretaria de Vigilância em Saúde, Departamento de Análise de Situação em Saúde. - Brasília : Ministério da Saúde, 2009. 84 p.

2. BRASIL. MINISTÉRIO DA SAÚDE. Secretaria de Vigilância em Saúde. Departamento de Análise de Situação em Saúde. Manual de Preenchimento das Fichas de Investigação do Óbito Materno/Ministério da Saúde, Secretaria de Vigilância em Saúde, Departamento de Análise de Situação em Saúde.-Brasília: Ministério da Saúde, 2011.

3. BRASIL. MINISTÉRIO DA SAÚDE. Secretaria de Vigilância em Saúde. Boletim Epidemiológico, 2020; 51(20).

4. CARVALHO PI, et al. Perfil sociodemográfico e assistencial da morte materna em Recife, 2006-2017: estudo descritivo. Epidemiol Serv Saúde, 2020; 29(1): e2019185.

5. COLLIER AY, MOLINA RL. Maternal Mortality in the United States: Updates on Trends, Causes, and Solutions. Neoreviews, 2019; 20(10): e561-e574.

6. CORTINHAS ABB, et al. Pré-eclâmpsia e mortalidade materna. Revista Caderno de Medicina, 2019; 2(1).

7. COSTA ES, et al. As principais causas de morte maternas entre as mulheres no Brasil. Revista Eletrônica Acervo Saúde, 2021; 13(1): e5826.

8. FALCÃO NHF, et al. Aspectos epidemiológicos da mortalidade materna no Brasil: A difícil tarefa de obtenção de dados reais. Brasília Med, 2011; 48(4): 403-408.

9. FEITOSA-ASSIS AI, SANTANA VS. Ocupação e mortalidade materna. Rev Saúde Pública, 2020; 54:64.

10. GINGREY JP. Maternal Mortality: A US Public Health Crisis. AJPH, 2020; 110(4): 462-464. 
11. MARTINS ACS, SILVA LS. Perfil epidemiológico de mortalidade materna. Rev Bras Enferm. 2018; 71(1): 725-31.

12. MORSE ML, et al. Mortalidade materna no Brasil: o que mostra a produção científica nos últimos 30 anos?. Cad Saúde Pública, 2011; 27(4):623-638.

13. NOUR NM. An Introduction to Maternal Mortality. Reviews in Obstetrics \& Ginecology, 2008; 1(2): 77-81.

14. OLIVEIRA AS, et al. Perfil epidemiológico dos casos de morte materna na cidade de Manaus por: causa, escolaridade e raça, no período de 2011 a 2015. Revista Eletrônica Acervo Saúde, 2019; (23): e424.

15. RUAS CAM, et al. Perfil e distribuição espacial da mortalidade materna. Rev Bras Saúde Mater Infant, 2020; 20(2): 397-409.

16. SACOOR C, et al. Health and socio-demographic profile of women of reproductive age in rural communities of Southern Mozambique. PLoS ONE, 2018; 13(2): e0184249.

17. SANTOS DR, et al. Mortalidade materna na população indígena e não indígena no Pará: contribuição para a vigilância de óbitos. Esc Anna Nery, 2017; 21(4): e20170161.

18. SCARTON J, et al. Perfil da Mortalidade Materna: Uma Revisão Integrativa da Literatura. Rev Fund Care, 2019; 11(3): 816-822.

19. VIANA RC, et al. Mortalidade Materna - uma abordagem atualizada. Com Ciências Saúde, 2011; 22(1): $141-152$.

20. VICTORA GC, et al. Maternal and child health in Brazil: progress and challenges. The Lancet, 2011; 377(9780): 18631876.

21. WHO. World Health Organization. Trends in Maternal mortality: 1990 to 2008. Estimates developed by WHO, United Nations International Children's Emergency Fund (UNICEF), United Nations Population Fund (UNFPA), and The World Bank, 2010.

22. WHO. World Health Organization. Trends in Maternal mortality: 1990 to 2015. Estimates developed by WHO, United Nations International Children's Emergency Fund (UNICEF), United Nations Population Fund (UNFPA), The World Bank, and the United Nations Population Division, 2015. 